served kinetochore protein required for cell cycle progression. Cell 86:275-285.

4.DeMarini, D.J., C.L. Creasy, Q. Lu, J. Mao, S.A. Sheardown, G.M. Sathe, and G.P. Livi. 2001. Oligonucleotide-mediated, PCR-independent cloning by homologous recombination. BioTechniques 30:520-523.

5.Good, L. and R.N. Nazar. 1992. An im proved thermal cycle for two-step PCR-based targeted mutagenesis. Nucleic Acids Res. 20:4934.

6.Kitagawa, K., D. Skowyra, S.J. Elledge, J.W. Harper, and P. Hieter. 1999. SGT1 encodes an essential component of the yeast kinetochore assembly pathway and a novel subunit of the SCF ubiquitin ligase complex. Mol. Cell. 4:21-33.

7.Lorenz, M.C., R.S. Muir, E. Lim, J. McElver, S.C. Weber, and J. Heitman. 1995. Gene disruption with PCR products in Saccharomyces cerevisiae. Gene 158:113117.

8.Papworth, C., J. Braman, and D.A. Wright. 1996. Strategies 9:3-4.

9.Picard, V., E. Ersdal-Badju, A. Lu, and S.C. Bock. 1994. A rapid and efficient one-tube PCR-based mutagenesis technique using $P f u$ DNA polymerase. Nucleic Acids Res. 22:2587-2591.

10. Rose, M.D., F. Winston, and P. Hieter. 1990. Methods in Yeast Genetics. CSH Laboratory Press, Cold Spring Harbor, NY

11.Sikorski, R.S. and P. Hieter. 1989. A system of shuttle vectors and yeast host strains designed for efficient manipulation of DNA in Saccharomyces cerevisiae. Genetics 122:1927.

12.Storici, F., L.K. Lewis, and M.A. Resnick. 2001. In vivo site-directed mutagenesis using oligonucleotides. Nat. Biotechnol. 19:773776.

13.Uetz, P., L. Giot, G. Cagney, T.A. Mansfield, R.S. Judson, J.R. Knight, D. Lockshon, V. Narayan, et al. 2000. A comprehensive analysis of protein-protein interactions in Saccharomyces cerevisiae. Nature 403:623627.

14. Winding, P. and M.W. Berchtold. 2001. The chicken B cell line DT40: a novel tool for gene disruption experiments. J. Immunol. Methods 249:1-16.

15.Zhang, Y., J.P. Muyrers, G. Testa, and A.F. Stewart. 2000. DNA cloning by homologous recombination in Escherichia coli. Nat. Biotechnol. 18:1314-1317.

This work was supported by Cancer Center Support grant no. CA 21675 from the National Institutes of Health and by the American Lebanese Syrian Associated Charities. Address correspondence to Dr. Katsumi Kitagawa, Department of Molecular Pharmacology, Mail Stop 230, St. Jude Children's Research Hospital, 332 N. Lauderdale St., Memphis, TN 38105, USA. email: katsumi.kitagawa@stjude.org

Received 11 March 2002; accepted 22 April 2002.
Katsumi Kitagawa and Rashid Abdulle St. Jude Children's Research Hospital Memphis, TN, USA

For reprints of this or any other article, contact Reprints@BioTechniques.com

\section{Deoxycholate-Based Method to Screen Phage Display Clones for Un- interrupted Open Reading Frames}

\section{BioTechniques 33:294-296 (August 2002)}

Phage display is a powerful technique broadly used in the expression of polypeptides with proper folding and thus function (9). Protein-3 (pIII, a minor protein) and protein- 8 (pVIII, the major protein) are two bacteriophage coat proteins that are generally used to display polypeptides as fusion proteins because their amino terminal regions are exposed on the phage particle surface and tolerate foreign polypeptide inserts. Such fusion proteins are usually expressed by phagemid constructs in the host cell and packed into the phage particle by the helper phage.

Most phage display studies involve libraries that are usually synthesized, semi-synthesized, or directly cloned from cDNA libraries. Unexpectedly, after panning against target proteins, a large percentage of isolated clones contain disrupted open reading frame (ORF), including in-frame stop codons, deletions, or frame-shift mutations $(3,5,6)$. Although the expression of non-ORF clones is much less efficient than that of ORF clones, as suggested by some studies (8), these non-ORF clones are usually enriched after several rounds of phage panning. One reason for the enrichment of non-ORF clones relates to the inhibitory role of pIII in phage superinfection. The infection titer of pIII-expressing bacterial cells by the helper phage is $2-3$ orders of magnitude lower than that of non-pIII parental cells (4). In phage display, the library sequences are usually inserted at the $5^{\prime}$-end of the gene III sequence in the phagemid. A phagemid containing an ORF sequence will produce protein III as a fusion and thus prevent the host cell from being infected by the helper phage, and the phagemid will less likely be packed into the phage particles. As each panning process requires a helper phage infection step, the abundance of non-favored ORF sequences will inversely correlate with the number of pannings.

To identify phage clones containing interrupted ORF, we describe here a simple method based on the sensitivity of the host cell to the ionic detergent deoxycholate. Expression of pIII has been shown to change the property of the bacterial outer membrane. As a consequence, the host bacteria appear to be sensitive to deoxycholate and more tolerant to certain colicins. In addition, leakage of periplasmic proteins such as $\beta$-lactamase can be detected (2). We were interested in determining if the deoxycholate sensitivity of pIII to host bacteria could be applied to the pIII-fusion expressed by phagemids.

We expressed the AHNP-Y peptide (YCDGFYACYMDV) (1) by phage display in the fusion to the $5^{\prime}$-end of the streptavidin core sequence (clone $\mathrm{H} 21$ ). Using $\mathrm{H} 21$ as a template, we introduced random sequences to replace AHNP-Y by PCR strategy. Briefly, a 5' random primer (Figure 1) and a $3^{\prime}$ streptavidin-specific primer were used to amplify the template, producing cDNAs of about $450 \mathrm{bp}$, which was subsequently cloned back into the phage vector pHage (Maxim Biotech, San Francisco, CA, USA). Several clones containing non-amber stop codons in the random peptide region are chosen with the original clone $\mathrm{H} 21$ and the empty vector pHage to test the deoxycholate-sensitivity of the host strain TG1 (Table 1 and Figure 2). In the TG1 strain, $\mathrm{H} 21$ and pHage express the full-length pIII as a fusion protein, while others express a short peptide when the translation is stopped by the non-amber stop codons.

The deoxycholate $[1.5 \% \quad(w / v)]$ plates were made by mixing $2 \mathrm{YT}$-agar 
Table 1. Growth of TG1 Bacteria Containing Different Phagemids on 2YT Plates with Ampicillin Only (Amp+) or Ampicillin Plus Deoxycholate (Amp+/DOC+)

\begin{tabular}{|cccc|}
\hline Phagemid & Stop Codon & Amp+ & Amp+/DOC+ \\
\hline- & - & No & No \\
H21 & No & Yes & No \\
T35H6 & Yes (TAA) & Yes & Yes \\
T33D5 & Yes (TAA) & Yes & Yes \\
\hline
\end{tabular}

and a pre-autoclaved $10 \%$ deoxycholate stock solution. All phagemid clones in TG1 were grown on a 2 YTagar plate overnight before being transferred to the special deoxycholate plate. We observed that all clones containing non-amber stop codons were able to grow on the deoxycholate-plate (Table 1). However, H21 and pHage, which expressed the pIII fusion, were sensitive to deoxycholate and did not grow on the deoxycholate plate (Table 1 and Figure 2). To make sure that all colonies were viable, we also simultaneously transferred a duplicate sample of each clone to a 2YT-ampicillin plate. All clones were able to form colonies after overnight incubation at $37^{\circ} \mathrm{C}$.

Since the expression of pIII fusion is regulated by the lac $Z$ promoter in the phage vector, glucose, an inhibitor of lacZ promoter, should repress the pIII fusion expression. We tested the sensitivity of clone $\mathrm{H} 21$ and pHage to deoxycholate in the presence of glucose
(Figure 2). As expected, after the pIII fusion expression was inhibited by glucose, both $\mathrm{H} 21$ and pHage clones in TG1 formed colonies on the deoxycholate-glucose plate ( $2 \%$ glucose). Interestingly, colonies on the glucose plate appear white, in contrast to the semi-transparent colonies on both 2YT and 2YT-deoxycholate plates. During our experiment, we also noticed that if picked from a glucose-plate, the $\mathrm{H} 21$ clone could grow on the deoxycholateonly plate, possibly because of the glucose carried over.

The phage library we constructed consisted of five amino acid residues randomly encoded by 64 codons including two non-amber stop codons. Since phages were prepared in an am ber-suppressor host TG1, the amber stop codon (TAG) was translated as glutamine. Theoretically, the possibility of having a non-suppressed stop codons (TGA or TAA) in any position is $15 \%\left(1-(62 / 64)^{5}=0.15\right)$. However,

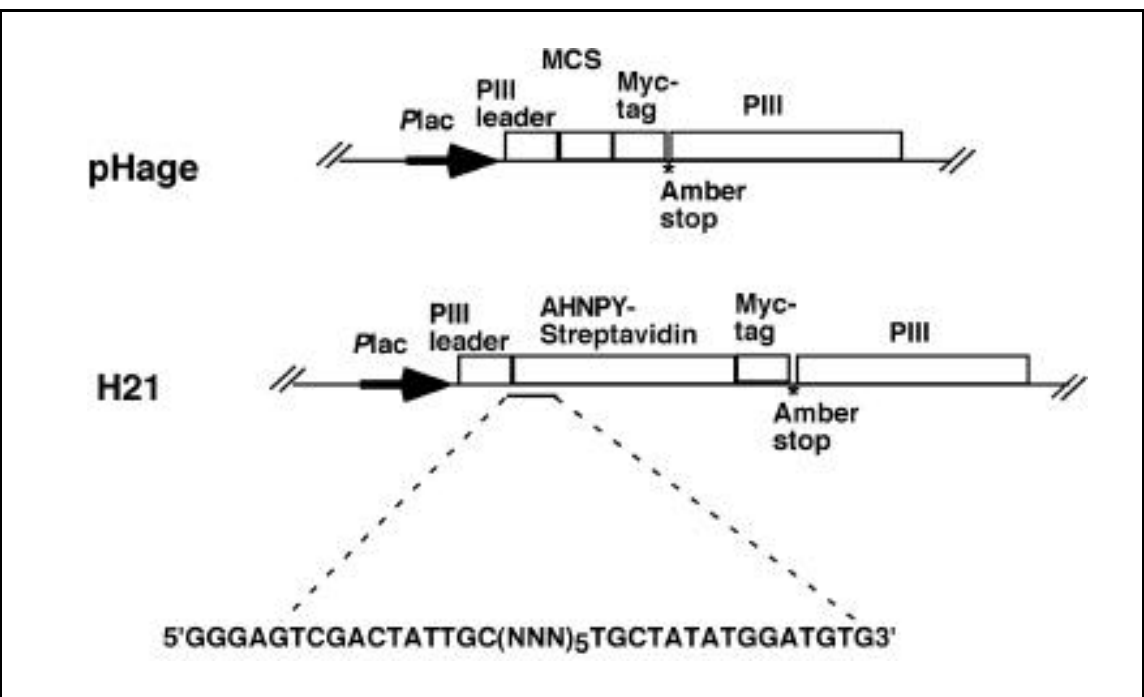

Figure 1. Schematic drawing of fusion protein region in the phagemids and the position of random primers used for phage display. pHage: the phage display vector using the lac Promoter (Plac). MCS: multiple cloning sites. H21: cDNA encoding AHNPY-Streptavidin was cloned into pHage before pIII. Both pHage and H21 contained an amber stop codon (TAG) between the Myc-tag and pIII. In an ambersuppressed strain such as TG1, both phagemids express pIII as a fusion protein. $n=\mathrm{G}, \mathrm{A}, \mathrm{T}$, or $\mathrm{C}$. 


\section{Benchmarks}

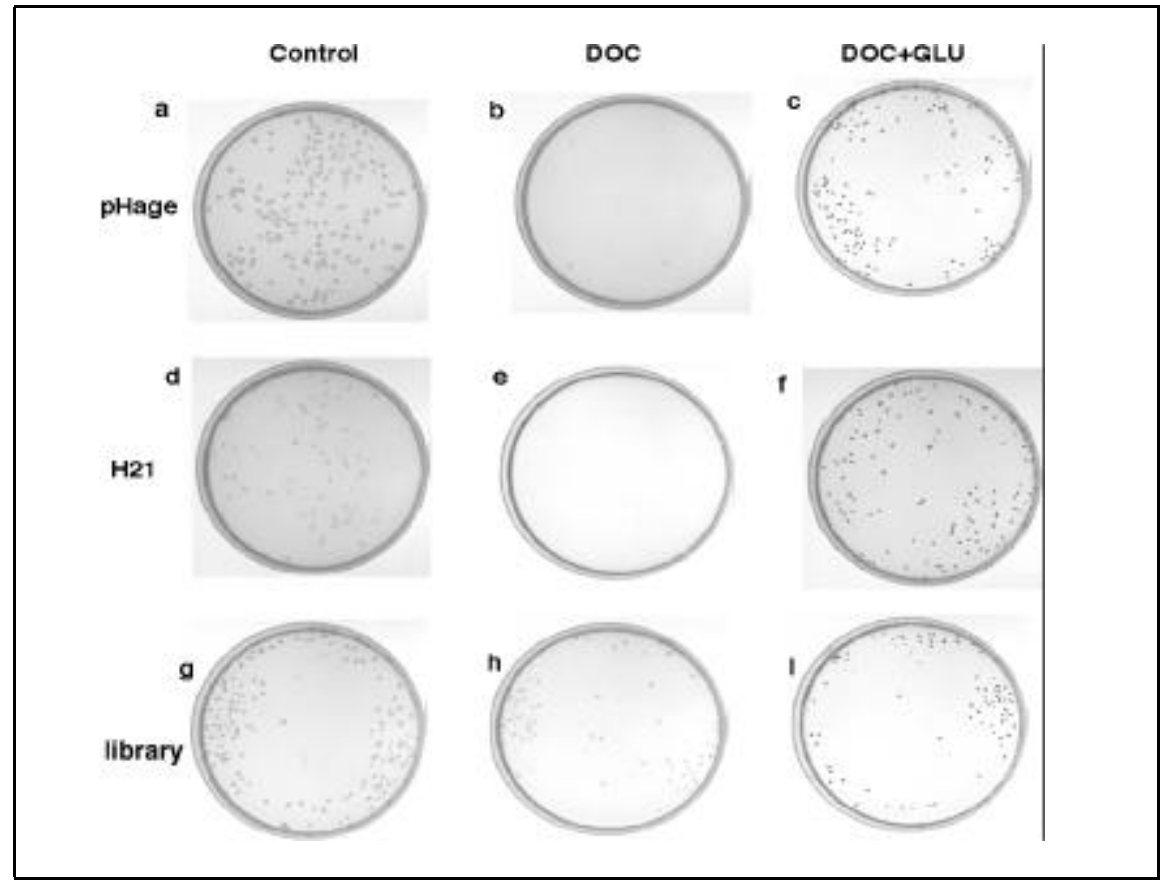

Figure 2. Effect of pIII-fusion expression on the growth of phagemid clones on deoxycholate plates. TG1 bacteria harboring pHage, $\mathrm{H} 21$, or the phage library were grown to $\mathrm{A}_{600}=1.0$ in $2 \mathrm{YT}$-amp either with (a, b, d, e, g, and h) or without (c, f, and i) $2 \%$ glucose. Bacterial culture was then diluted 10-5, and $100 \mu \mathrm{L}$ of the diluted culture were plated on 2YT, 2YT plus DOC, or 2YT plus both DOC and GLU as indicated. All plates contained ampicillin $(100 \mu \mathrm{g} / \mathrm{mL})$. Colony numbers of DOC+GLU plates are not comparable with those of other plates because different bacterial culture was used. The DOC+GLU plates were included to test qualitatively, not quantitatively, the ability of bacteria to form colonies. DOC: deoxycholate, GLU: glucose.

the number of colonies formed on the deoxycholate plate was about $39 \%$ of that on the non-deoxycholate plate (Figure 2), suggesting that the library contains more non-ORF clones than expected (15\%). It is worth mentioning that we prepared the library in the presence of glucose. Without glucose to suppress the expression of pIII fusion, a much higher percentage of non-ORF clones would be observed. In addition, it is likely that the amber codon was not fully suppressed in strains like TG1.

Several protein translation theories, including translational re-coding, readthrough of stop codons, and ribosomal slippage, have been raised to explain the display of non-ORF clones (7). It is not surprising that in many cases these nonORF clones are true clones that really bind to the target proteins. However, because of the low expression of the nonORF clones, further characterization of these clones is not a favorable option. Our results show that the deoxycholate plate can be used to identify a non-ORF phagemid. Although DNA sequencing is necessary to confirm the sequences of interesting clones, the deoxycholate method discussed here is very useful in screening phagemid clones to exclude possible ORF-disruption clones. Currently, restriction enzyme digestion is used to identify deletions, while stopcodon clones are not revealed until DNA sequencing. Both methods involve bacterial culturing, plasmid preparation, expensive enzyme reactions, and electrophoresis, while our method is simple, quick, and inexpensive.

\section{REFERENCES}

1.Berezov, A., H.T. Zhang, M.I. Greene, and R. Murali. 2001. Disabling erbB receptors with rationally designed exocyclic mimetics of antibodies: structure-function analysis. J. Med. Chem. 44:2565-2574.

2.Boeke, J.D., P. Model, and N.D. Zinder. 1982. Effects of bacteriophage f1 gene III protein on the host cell membrane. Mol. Gen. Genet. 186:185-192.

3.Carcamo, J., M.W. Ravera, R. Brissette, O. Dedova, J.R. Beasley, A. Alam-Moghe, C. Wan, A. Blume, and W. Mandecki. 1998. Unexpected frameshifts from gene to expressed protein in a phage-displayed peptide library. Proc. Natl. Acad. Sci. USA 95:11146-11151.
4.Duenas, M. and C.A. Borrebaeck. 1995. Novel helper phage design: intergenic region affects the assembly of bacteriophages and the size of antibody libraries. FEMS Microbiol. Lett. 125:317-321.

5.Goldman, E., M. Korus, and W. Mandecki. 2000. Efficiencies of translation in three reading frames of unusual non-ORF sequences isolated from phage display. FASEB $\mathrm{J}$. 14:603-611.

6.Jacobsson, K. and L. Frykberg. 1996. Phage display shot-gun cloning of ligand-binding domains of prokaryotic receptors approaches 100\% correct clones. BioTechniques 20:10701081.

7.Parker, J. 1989. Errors and alternatives in reading the universal genetic code. Microbiol. Rev. 53:273-298.

8.Schier, R., J. Bye, G. Apell, A. McCall, G.P. Adams, M. Malmqvist, L.M. Weiner, and J.D. Marks. 1996. Isolation of high-affinity monomeric human anti-c-erbB-2 single chain Fv using affinity-driven selection. J. Mol. Biol. 255:28-43.

9.Smith, G.P. 1985. Filamentous fusion phage: novel expression vectors that display cloned antigens on the virion surface. Science 228:1315-1317.

We thank Mark Richter for his comments on the manuscript. This work was supported by grants from Abramson Institute to M.I.G. and an award from University of Pennsylvania Research Foundation to R.M. and H.Z. Address correspondence to Dr. Hongtao Zhang, Department of Pathology and Laboratory Medicine, University of Pennsylvania School of Medicine, Philadelphia, PA 19104, USA. e-mail: zhanghon@ mail.med.upenn.edu

Received 12 March 2002; accepted 25 April 2002.

B. Li, H. Zhao, Q. Liu, R. Murali, M.I. Greene, and H. Zhang University of Pennsylvania School of Medicine Philadelphia, PA, USA

For reprints of this or any other article, contact Reprints@BioTechniques.com 